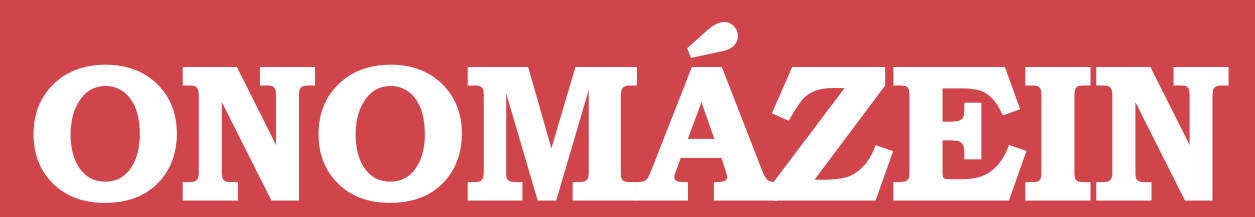

Revista de lingüística, filología y traducción
PONTIFICIA UNIVERSIDAD

Camino, Base y Manera en bilingües de español y sueco: efectos de una segunda lengua en los patrones de expresión del movimiento de una primera lengua

Path, Ground and Manner in Spanish-Swedish

bilinguals: the effects of a second language in the patterns for motion encoding of a first language

\title{
Alejandra Donoso
}

Universidad de Estocolmo

Suecia

\section{(C) $($ (i) $\ominus$}




\section{Resumen}

En este estudio se investiga cómo los hablantes bilingües de español y sueco expresan eventos de movimiento en su lengua heredada o lengua materna: español. La premisa inicial es que la conceptualización habitual de eventos no sólo sirve como base para el uso de una segunda lengua (L2) (Flecken y otros, 2014: 51), sino también para el uso de una lengua materna (LI) en sujetos bilingües (Bylund y Jarvis, 2011). Tomando como punto de partida estudios anteriores sobre bilingüismo y adquisición de segundas lenguas, nos proponemos averiguar cómo los patrones tipológicos para la codificación del movimiento de una L2 (una lengua de marco satelital) influyen en la codificación del movimiento de una Lı (una lengua de marco verbal). En este caso se trata de una Li con la cual los sujetos estudiados han ido perdiendo contacto desde su llegada a Suecia. Considerando este hecho, y basándonos en la premisa de que haber perdido contacto con el entorno de la LI tiene un impacto en cuanto a cómo los eventos de movimiento son descritos en una LI (Bylund, 2009), aspiramos a caracterizar la conceptualización de eventos de movimiento de dos grupos de bilingües, en total 31 sujetos, y determinar los diferentes fenómenos de transferencia que podrían afectar su producción oral. Con este fin hemos comparado narraciones producidas por los bilingües con dos grupos de control (monolingües de sueco y español), estableciendo las divergencias que estas presentan en torno a la entrega de los componentes semánticos básicos de un evento: Manera, Camino y Base. Nuestros hallazgos nos permiten concluir que tanto la edad de adquisición de la L2 así como el tiempo de residencia en el entorno de esta lengua han afectado los patrones de conceptualización del movimiento en la Lı.

Palabras clave: español como lengua heredada; adquisición de segundas lenguas; transferencia conceptual; "pensar para hablar”; eventos de movimiento.

\section{Abstract}

This study addresses how Spanish-Swedish early and late bilinguals express motion events in their inherited language or first language: Spanish. We draw on the idea that the habitual conceptualization of events also underlies both L2 usage (Flecken et al., 2014: 51) and L1 usage in bilinguals (Bylund and Jarvis, 2011). Drawing both on studies about second language acquisition and bilingualism we aim to study how the typological patterns for mo- 
tion encoding of the L2 (a satellite-framed language) may impact on motion encoding in the LI (a verb-framed language), which the group under study has had but a reduced contact with while growing up in Sweden. Considering this fact and starting off from the assumption that an early age of break with the LI environment has an impact on how motion events are described in an LI (Bylund, 2009), we aim to outline the conceptualization of motion events of two groups of bilinguals, in all 31 subjects, and the different sorts of transfer phenomena that may affect their speech. Oral narratives produced by the bilinguals have been compared to two control groups of Spanish and Swedish monolinguals and then been examined in order to analyze their conflation patterns regarding Manner of motion, Path and Ground information. Our results have lead us finally to conclude that both the individuals' age of second language acquisition and their length of residence in the L2 environment have affected their L1 conceptualization patterns.

Keywords: Spanish as a heritage language; L2 acquisition; bilingualism; conceptual transfer; thinking for speaking, motion events. 


\section{Introducción}

Muchos estudios centrados en investigar la expresión del movimiento en las diversas lenguas del mundo han llegado a conclusiones decisivas respecto del impacto que los patrones característicos de estructura y entrega de este tipo de información tienen para la conceptualización en general y, en particular, para la conceptualización en hablantes bilingües, tema que aquí nos compete. Sabido es que hablantes de lenguas distintas recurren sistemáticamente a patrones específicos para estructurar la información relativa al movimiento (Talmy, 1985, 2000; Slobin y Bocaz, 1988; Slobin, 1991, 1996, 1997; Naigles y Terrazas, 1998; Hendriks y Hickmann, 2011). Por lo demás, estas diferencias han demostrado influir en la producción oral de los hablantes desde la infancia e incluso desde antes de los 2 años de edad (Choi y Bowerman, 1991; Bowerman, De León y Choi, 1995), y ser, asimismo, de difícil restructuración en el proceso de adquisición de una segunda lengua (Flecken, Von Stutterheim y Carroll, 2014). En innumerables casos, los aprendientes de una segunda lengua (L2) deben enfrentarse al desafío de no tan solo aprender los aspectos formales de la L2 para hablar de movimiento, como, por ejemplo, el uso de determinadas preposiciones (cf. Larrañaga y otros, 2012), sino también el de adquirir las reglas y principios que guían la estructura de la información en la lengua meta (cf. Donoso y Bylund, 2015: 234), principios que en muchos casos se permean desde la L1 a la L2 (Von Stutterheim, 2003; Cadierno, 2004; Hohenstein, Eisenberg y Naigles, 2006; Schmiedtová y Flecken, 2008; Hendriks y Hickmann, 2011; Donoso, 2014; Donoso y Bylund, 2015).

Similar desafío encontrarán aquellos sujetos bilingües para quienes la Lı represente un sistema lingüístico al que han debido agregar uno distinto, tal vez, incluso, desde una edad temprana. Es más, y lo cual puede resultar desventajoso: mientras más temprano hayan adquirido la L2, menos cercanos podrían volverse a la norma nativa de la L1, particularmente en aquellos casos en que el contacto con su lengua materna, por una u otra razón, se ha visto reducido (Hakuta y D’Andrea, 1992; Silva-Corvalán, 1994; Bylund, 2009). Su dominio de la L2, especialmente en aquellos casos en que la L2 constituye una lengua dominante, puede exceder, no obstante, al dominio que tienen de su lengua materna.

Diversos estudios enfocados en la variable 'edad de adquisición de la L2' (age of onset of L2 acquisition) avalan la tesis anterior, y han demostrado que, por un lado, una edad temprana de adquisición de la Lz es decisiva en cuanto al nivel de proficiencia que pueda alcanzarse en esta lengua (DeKeyser, 2000; Abrahamsson y Hyltenstam, 2009) y que, por otro, este aspecto, sumado a un contacto reducido con la LI, incide de forma negativa en la retención de aquellos componentes lingüísticos de la Lı que se desarrollan antes de la pubertad, como la proficiencia léxica y la morfosintáctica (Montrul, Foote y Perpiñán, 2008). Caso análogo está constituido por los patrones de conceptualización de eventos de movimiento (EM), ámbito de la codificación lingüística relativamente menos estudiado en el español de los grupos que aquí nos interesan: el de los bilingües tardíos, vale decir, de aquellos que han adquirido el sueco 
como L2 en la adolescencia o en edad adulta, y el de los bilingües tempranos, para quienes el español es una lengua a la que han estado expuestos en su entorno familiar desde la infancia (pudiendo considerarse esta como una lengua heredada), a la par que han adquirido el sueco fuera de casa. A saber, los patrones de conceptualización tenderían a distanciarse de la norma nativa de la LI, particularmente en aquellos casos en que los bilingües han adquirido una $L 2$ antes de la pubertad, indicando con ello que los patrones adquiridos mediante la LI no se mantendrían estables en el proceso de adquisición de una L2 y que, por consiguiente, la L2 podría influir en los procesos de conceptualización en la LI (Bylund, 2009).

Tomando como punto de partida lo mencionado hasta aquí, en este estudio nos interesa averiguar cuáles son las características que exhibe un conjunto de narraciones descritas en español por usuarios bilingües de español L1 / sueco L2, cuya edad de adquisición de la L2 varía, y cuya pérdida de contacto con la Lı se produjo a distintas edades. Tomando en cuenta que aprender una lengua ya pasada la pubertad difícilmente conduce a su maestría absoluta (a diferencia de lo que puede ocurrir con aquellas lenguas que se aprenden en la infancia) y que dicha L2, en consecuencia, puede no ejercer un influjo en la L1 (Hohenstein y otros, 2006: 251), nos preguntamos de qué forma los patrones de expresión de EM característicos del sueco como L2 tendrán un impacto en las descripciones en español L1, sumándonos con ello a la idea de que los patrones del "pensar para hablar" (Slobin, 1996) de una L2 pueden influir en las descripciones de los hablantes bilingües, aunque no de aquellos sujetos bilingües que han adquirido la L2 tras la pubertad. Nos interesan, en particular, los usos novedosos del grupo aquí examinado, los giros idiomáticos para hablar del movimiento y, en general, aquello que puede rastrearse a alguna de las lenguas de los sujetos, pero que bien puede llegar a constituir una suerte de interlengua o sistema que se halla entre la Lı y la L2, diferenciándose con ello de ambas lenguas.

\section{Expresión del movimiento: aspectos contrastivos generales y específicos (sueco y español)}

A modo general, un EM ha sido definido como un suceso en que un objeto animado o no animado ('Figura') se desplaza ('Camino') de un lugar a otro, pudiendo abarcar tanto un origen como un destino ('Base'). Opcionalmente incluye la alusión a una 'Causa' y una 'Manera' de moverse. De acuerdo a cómo las lenguas representan el componente de Camino, suelen ser divididas en dos grupos (Talmy, 1985), las de marco verbal, o lenguas-V, que lexicalizan el Camino en el verbo principal, como ocurre con el español y las lenguas romances en general, y las de marco de satélite, o lenguas-S, que, como el sueco, lo expresan por medio de una partícula satelital.

A saber, las descripciones del Camino en sueco suelen estar representadas por la estructura 'verbo + partícula satelital' (Donoso, 2013, 2014), como en la oración mannen gick ut ("el hombre fue afuera”, traducción literal), mientras que en español el Camino suele aparecer le- 
xicalizado en un verbo principal (i. e., 'el hombre salió'). Asimismo, el Camino en sueco aparece frecuentemente complementado por alusiones al trasfondo físico (la Base) en que ocurren los desplazamientos, pudiendo apreciarse incluso un al to número de complementos de lugar unidos a un mismo verbo, el que puede ser tanto un verbo de dirección inherente (como p. ej. el verbo att komma, 'venir') como un verbo de manera con desplazamiento (p. ej. el verbo att springa, 'correr') o un verbo de manera sin desplazamiento, como ocurriría en el ejemplo siguiente con el verbo att hoppa ('saltar'):

(1) hunden hoppade ut genom fönstret och bort mot skogen.

\begin{tabular}{|c|c|c|c|c|}
\hline $\begin{array}{l}\text { hund-en } \\
\text { perro- }\end{array}$ & $\begin{array}{l}\text { hoppa-de } \\
\text { saltar-PST }\end{array}$ & $\begin{array}{l}\text { ut } \\
\text { hacia.afuera.ADV }\end{array}$ & $\begin{array}{l}\text { genom } \\
\text { a.través.de.PREP }\end{array}$ & fönstr-et \\
\hline DEF & & & & DEF \\
\hline FIGURA & MANERA & CAMINO & CAMINO & BASE \\
\hline och & bort & mot & skog-en & \\
\hline $\mathrm{y}$ & lejos.ADV & hacia.PREP & bosque-DEF & \\
\hline & CAMINO & CAMINO & BASE & \\
\hline
\end{tabular}

"El-perro saltó hacia-afuera por la-ventana y lejos hacia el-bosque".

En sueco, el esquema de ‘verbo + partícula satelital + un posible destino’ representa una alternativa de uso frecuente para la expresión de eventos de movimiento (Donoso, 2013)․․ Su uso está relativamente convencionalizado en la lengua vernácula y coincide, aparentemente, con dos factores: el que los verbos de movimiento en esta lengua-S (independientemente de si

1 Es importante notar que verbos direccionales como ir, regresar, venir, bajar, subir o volver, suelen aparecer frecuentemente en español con complementos que señalan el Origen y la Meta del desplazamiento, al menos en lo que compete a la variante mexicana (Ibáñez, 2002). No obstante, hemos de enfatizar que en sueco - a diferencia de lo que ocurre con el español- tales complementos tienden a aparecer con cualquier tipo de verbo (Donoso, 2013; Sjöström, 1990), cuestión análoga a lo que ocurre en otras lenguas germánicas, tal y como queda demostrado en los estudios de, por ejemplo, Carroll y otros (2004), Bylund (2009), cuyos resultados dan prueba de la alta recurrencia de complementos de destino en la descripción de eventos de movimiento. Por esta razón, y tomando en cuenta que estas características propias a una lengua-S pueden influir en las narraciones de sujetos cuya Li corresponde al grupo de las lenguas-V, aclaramos que se ha estudiado los verbos dependiendo del grado de predicación propuesto por Ibáñez (2002), en los grupos (1)-(3) que se señalan a continuación, añadiendo las especificaciones descritas por Morimoto (2001) al grupo 3: (1) aquellos verbos que cubren todo el proceso de cambio de lugar delimitado por un punto de partida y arribo (p. ej. en verbos como "ir"), (2) aquellos que perfilan solo una parte del proceso, ya sea el punto de partida o la Meta ("partir", "subir"), y (3) aquellos que contienen Manera, ya sea en conjunto con un sentido de desplazamiento (“correr”) como sin él ("saltar"). 
contienen o no Manera) exijan, comúnmente, que la trayectoria del movimiento sea expresada fuera del verbo principal (Sjöström, 1990) y con el hecho de que el sueco es una lengua sin aspecto gramaticalizado (i. e., no contiene las formas gramaticales del presente continuo de lenguas como el español o el inglés), fenómeno que coexistiría con una tendencia a presentar los eventos de movimiento de forma holística tal y como sucede en otras lenguas (por ejemplo el alemán) que no cuentan con la distinción de aspecto gramaticalizado² (Carroll y otros, 2004; Bylund, 2009). A ello se suma el que la Meta expresada mediante el objeto en cuestión no resulte redundante en el habla, como podría ocurrir en español. Compárese las siguientes oraciones, tomadas de dos sujetos que narran exactamente el mismo evento de movimiento, el primero en sueco (2) y el segundo en español (3):

(2) Han föll ner på marken

$\begin{array}{lllll}\text { han } & \text { föll } & \text { ner } & \text { på } & \text { mark-en } \\ \text { él } & \text { caer.PST } & \text { hacia.abajo.ADV } & \text { en.PREP } & \text { suelo-DEF } \\ \text { FIGURA } & \text { MOVIMIENTO } & \text { CAMINO } & \text { BASE } & \text { BASE }\end{array}$

"él cayó hacia-abajo en el-suelo"

(3): Se cayó

En el caso de (3) "se cayó”, para la narración en español nativo, la dirección ('hacia abajo') está contenida en el verbo "caer"; la Base, por otro lado, puede aparecer expresada fuera del verbo en caso de que el hablante decida perfilar la Meta, de lo contrario, puede recuperarse a partir de los elementos contextuales; mientras que en la opción (2), aparecen tanto el Camino como la Base, representando una alternativa posible, por lo demás plenamente aceptada, en sueco. De esta forma, dos de los componentes semánticos que conforman todo evento de movimiento (Camino y Base), suelen aparecer de forma explícita en sueco (Donoso, 2013). En español, por el contrario, tales descripciones de movimiento con un sentido direccional inherente (para más detalle, pueden consultarse Ibáñez (2002) y Zubizarreta y Oh (2007)) suelen estar regidas por el verbo principal (el que ya contiene información relativa al Camino, como en el caso de los verbos "subir" o "entrar"), de tal manera que la indicación de destino o Meta puede ser elidida. La presencia de una partícula satelital³, por otra parte, no se hace relevante

2 A lo largo de este trabajo se ha usado la denominación de aspecto gramaticalizado para hacer referencia a aquellos eventos de movimiento que expresan una actividad en curso por medio del presente continuo (p. ej. en "dos monjas van caminando hacia una iglesia").

3 El concepto de "satélite" de Talmy no deja de ser controvertido (ver p. ej. las críticas expuestas por Beavers, Levin y Tham (2010)). En este trabajo, operaremos con esta distinción para referirnos a elementos que conllevan una dirección, tales como, por ejemplo, los adverbios arriba, abajo o adentro, preposiciones tales como hacia o a, y deícticos como aquí o allí. 
por la casi ausencia de esta categoría en español. En consecuencia, la información relativa al movimiento y el Camino suele aparecer expresada en español mediante un verbo 'solitario', un bare verb usando las palabras de Slobin (1996).

Por otra parte, la Manera (aspecto del movimiento frecuentemente expresado en las lenguas-S) suele ser expresada en sueco al interior del verbo principal, mientras que en español, si es que se expresa, suele aparecer en un verbo en gerundio (compárese: hon dansade in $i$ huset, 'ella bailó para-adentro en/a la-casa' con sentido direccional, con la oración "ella entró bailando en la casa”, en que la Manera aparece expresada en un verbo en gerundio) o bien en adverbios o locuciones de distinto tipo. El sueco, adhiriendo al marco de las lenguas-S, presenta un abanico mayor de verbos de manera que las lenguas romances (Tegelberg, 2002); asimismo, ostenta la facultad de poder unir todo tipo de verbos de manera ${ }^{4}$ a diversas partículas satelitales y complementos de Base (Donoso, 2013), tal y como ocurre con los verbos "correr" y "caminar" (Manera con desplazamiento) o con los verbos del tipo de "temblar" (Manera sin desplazamiento), de acuerdo a la clasificación de Morimoto (2001). Los hablantes de español, en cambio, al codificar el componente de Manera fuera del verbo principal, convertirían su expresión en el habla en una alternativa opcional (Jovanovic y Martinovic-Zic, 2004; Slobin, 2004).

Como hemos visto hasta aquí, las diferencias relativas a la codificación y entrega del Camino, la Manera y la Base tienen un impacto decisivo en cuanto a los elementos de un EM que los hablantes suelen escoger y relacionar a la hora de comunicarse. Estudios específicamente enfocados en las diferencias contrastivas entre grupos de monolingües de español y sueco, como los de Bylund (2009) y Donoso (2013), indican asimismo que tales patrones de expresión de EM influyen en los patrones de conceptualización, vale decir, los hablantes monolingües de sueco:

a) prestan más atención que los monolingües de español a la Manera, lo cual se ve reflejado en una mayor frecuencia de alusiones a este componente (ya sea por medio de verbos de manera o expresiones adverbiales de Manera);

4 Como ya se ha hecho notar en diversos estudios (ver p. ej. el trabajo de Hijazo-Gascón y otros (2013)), definir un verbo de manera es complejo. Ibáñez (en proceso) propone definir este término en cuanto a lo que denomina como "formante lexicalizado", distinguiendo entre manera del desplazamiento ("correr", "caminar"), manera de la trayectoria (“circular", "rondar"), manera de la Figura en ausencia de desplazamiento ("bailar", "girar") y manera con relación al fondo ("volar", "nadar"). En el presente artículo, nos referimos a los verbos de manera tomando en cuenta, en primer lugar, el movimiento de la Figura cuando se desplaza; asimismo, y dado que tanto la realización argumentativa de la Manera como su expresión en elementos verbales no es equivalente entre las lenguas que nos competen, cuando estudiamos este componente lo hacemos por medio de la consideración a un grupo vasto de verbos de movimiento, tal y como se ha propuesto en Morimoto (2001). 
b) presentan una mayor alusión a los puntos finales de los eventos, manifestándose ello en una alta frecuencia de partículas satelitales y un mayor número de complementos de destino.

Tomando como punto de partida estos resultados, en este estudio avanzamos que los bilingües tempranos estarán guiados por los patrones de estructuración y entrega de la información específicos de su L2 (el sueco), influyendo estos en el grado de desarrollo con el que los distintos componentes de un EM serán expresados en el habla. Caso opuesto estará constituido por los bilingües tardíos, quienes no se verán afectados por la L2 en la misma medida. A continuación haremos algunas aproximaciones respecto de cómo la conceptualización y expresión de eventos de movimiento ha sido estudiada concretamente en sujetos bilingües a partir de distintas premisas en torno a los fenómenos de transferencia y convergencia.

\section{Transferencia y conceptualización de eventos en sujetos bilingües}

Los fenómenos de transferencia, conocidos a través de los términos 'transferencia', 'interferencia' o 'influencia interlingüística', han sido ampliamente estudiados no solo en cuanto a la transferencia de aspectos formales, sino también conceptuales (Treffers-Daller, 2012). El fenómeno de la transferencia inversa (reverse transfer) o transferencia desde la L2 a la LI es uno que, sin embargo, se ha venido estudiando solo en años recientes. Treffers-Daller y Sakel (2012) señalan al respecto que investigar sujetos multilingües puede ayudarnos a entender con mayor precisión las similitudes y diferencias entre los fenómenos de convergencia y transferencia (2012: 4-5). La convergencia como fenómeno colindante a la transferencia implica que el sujeto bilingüe ha creado una interlengua que se halla entre la L1 y la L2, diferenciándose con ello de ambos sistemas; la transferencia inversa, por otro lado, adjudica las desviaciones observables en la LI al influjo de los patrones lingüísticos propios de la L2.

En general, y refiriéndonos, en primer lugar, a la díada $L 1>L 2$, los resultados de los estudios sobre la transferencia de patrones conceptuales parecen no ser del todo conclusivos en cuanto al impacto que la Lı podría tener en la adquisición de una L2 (Treffers-Dallers, 2012). En el caso de la adquisición de una L2 de marco verbal, estudios anteriores, como los de Cadierno (2004), Navarro y Nicoladis (2005), Cadierno y Ruiz (2006), Hijazo-Gascón (2011), Donoso (2014) y Donoso y Bylund (2015), han demostrado que ciertos aspectos de la L2 (tales como la entrega del Camino en un verbo de desplazamiento) pueden ser adquiridos en edad adulta sin mayor dificultad. No obstante, Larrañaga y otros (2012) señalan que ciertas dificultades prevalecerían, tales como la de usar verbos de Manera uniéndolos a predicados télicos (característica propia de las lenguas-S), incluso en aquellos casos en que se atraviesa un límite físico, cuestión que en ciertos casos no resulta agramatical, pero sí inapropiada (véase por ej. Ia oración 'corre en un banco' con sentido direccional en Larrañaga y otros (2012: 14)). De 
acuerdo a Athanasopoulos (2011: 37), citado en Treffers-Dallers (2012), determinadas variables lingüísticas y socioculturales, tales como el nivel específico de proficiencia del aspecto lingüístico investigado, la proficiencia lingüística general, el ambiente de interacción (si es que los sujetos se encontraban en el ámbito de la lengua meta al momento de ser estudiados), la edad de adquisición (EA) de la $L 2$, el nivel de contacto (NC) con la L2 y el tiempo de residencia (TR) en el país de la lengua meta, tendrían un impacto ineludible en los patrones del "pensar para hablar" en la L2.

Lo mismo sería efectivo a la inversa, vale decir, ciertas variables lingüísticas y socioculturales tendrían un impacto en la evolución de la $L$ ( (díada $L 2>L 1$ ), ya sea favoreciendo su expansión en aquellos casos en los que el adquirir una L2 se suma al conocimiento preexistente en la Lı (bilingüismo aditivo) o bien contribuyendo a su pérdida, fenómeno conocido como atrición (Köpke y Schmid, 2004). Como exponíamos más arriba, si bien el influjo de la L2>L1 ha comenzado a ser sistemáticamente estudiado dentro de las últimas décadas (Cook, 2003), el del impacto de los patrones conceptuales es uno que más bien ha prosperado recientemente en trabajos como los de Bylund (2011) y Brown y Gullberg (2011), siendo particularmente novedoso en lo referente a los patrones conceptuales para hablar de Manera, Base y Camino, como veremos a continuación.

A modo general, los estudios llevados a cabo en sujetos bilingües han aportado unos resultados de difícil interpretación: la dificultad radica, grosso modo, en que ciertos comportamientos lingüísticos representan una colección de patrones mixtos. Algunos estudios efectuados dentro del abanico representado por las distinciones tipológicas en cuanto a la lexicalización del Camino (tipología de Talmy), dan cuenta de que los bilingües parecerían hallarse entre los patrones de conceptualización de su L1 y su L2. Así lo demuestran Hohenstein y otros (2006), cuyo estudio sobre transferencia bidireccional en bilingües de español LI/ inglés L2 ha reportado que los bilingües entregan más verbos de manera que los monolingües de español, y más verbos de trayectoria (o verbos de desplazamiento) que los monolingües de inglés (lengua-S). Su estudio, cuyo objetivo era investigar elecciones lexicales y estructuras gramaticales en EM, pone a prueba la tesis de que las especificidades de las estructuras gramaticales y el léxico de una lengua u otra pueden ejercer una influencia bidireccional, y que la ambigüedad estructural de la lengua sensible a sufrir una transferencia puede explicar en parte las elecciones léxicas y gramaticales de sus usuarios; vale decir, si ambas lenguas presentan las mismas estructuras/ léxico habrá, entonces, transferencia. Tomando estos hallazgos en cuenta, Bylund (2011: 7) señala que los autores han sido cuidadosos en cuanto a cómo interpretarlos, evitando clasificarlos como un constructo entre L1 y L2. No obstante, y como indican Jarvis y Pavlenko (2008), al parecer los bilingües estudiados se habrían vuelto más sensibles a los componentes semánticos de Manera y Camino como resultado del uso de ambas lenguas (Bylund, 2011). 
Por otro lado, Bylund (2011), adhiriendo a los estudios abocados a investigar la conceptualización de eventos en sujetos bilingües a partir de las distinciones témporo-aspectuales (para más detalles sobre este marco teórico consultar por ej. Von Stutterheim y Nüse (2003)), concluye que sus sujetos estudiados presentan, por un lado, patrones de segmentación (i. e., mención a los distintos segmentos que podrían constituir un EM) que se encuentran entre la L1 y la L2 y, por otro, estrategias de anclaje temporal con las que se acomodan a la respectiva lengua en uso. Sus hallazgos indican, además, que estos patrones no varían en razón de la edad de adquisición de la L2, ni tampoco frente a las variables tiempo de residencia en el entorno de la L2 ni nivel de contacto con la LI (aspecto evaluado de acuerdo al porcentaje estimado de uso diario de la Lı declarado por cada informante). En síntesis, sus resultados validan, aunque solo en cierta medida, la hipótesis de la convergencia conceptual, dando también lugar a nuevas preguntas, tales como si los patrones mixtos de segmentación — dada la variedad intragrupal encontrada - no podrían ser un reflejo de otros fenómenos, tales como los de cambio conceptual ('patrones de la LI basados en la L2') o transferencia conceptual de la LI ('patrones conceptuales de la L2 basados en la LI'), dependiendo del grupo monolingüe al cual los sujetos estudiados se aproximan (2011: 32).

En la misma línea, Bylund y Jarvis (2011), tras investigar la expresión de EM en español de un grupo de 40 bilingües de español L1 / sueco L2 con distintas edades de adquisición, plantean la hipótesis de que los sujetos estudiados presentarán una tendencia a enfocarse en los eventos de movimiento de forma holística (i. e., refiriéndose a los puntos finales [PF] en mayor medida que los monolingües de español) tal y como sucede en las lenguas sin marca aspectual (como el alemán y el sueco). Sus hallazgos indican que, efectivamente, aquellos hablantes que llevan más tiempo sin tener contacto con el habla hispana (lengua rica en distinciones aspectuales) tienden a entregar más PF, expresando de esta forma los eventos como si estos estuvieran acabados, alejándose con ello de la forma típica en que los eventos de movimiento son expresados en español nativo, vale decir, como eventos que se expresan desde su desarrollo y progresión interna. Según los autores, la activación de enfoques temporales de este tipo depende notablemente de la recurrencia con que estos son usados y reforzados en el habla, lo cual implicaría que la probabilidad de que un hablante acceda a un determinado tipo de esquema temporal en desmedro de otro podría cambiar con el paso del tiempo. Esto se volvería palpable, atendiendo a Bylund y Jarvis (2011: 50), en aquellos casos en que la naturaleza de los eventos a los que la persona se ve expuesta cambian.

\section{El presente estudio 4.1. Objetivos e hipótesis}

Como se desprende de los apartados anteriores, si bien muchos de los estudios que competen a sujetos bilingües dan cuenta de un influjo importante de la L2 en la LI, bastante queda 
por averiguar sobre cómo la conceptualización de eventos en la Lı puede verse influida por los patrones de entrega de información de movimiento propios de una L2. Investigar el campo de la conceptualización es una tarea compleja, puesto que va más allá del examen de los aspectos puramente formales. Este estudio pretende, en consecuencia, proporcionar nuevos datos sobre la configuración bilingüe en lo que a la conceptualización de eventos se refiere, enfocándonos, particularmente, en la entrega de información relativa a la Manera, el Camino y la Base. Asimismo, aspira a discutir los resultados con relación a tres factores que hasta ahora la literatura ha clasificado como eventualmente determinantes para la adquisición de una L2 y, eventualmente, para los casos de adquisición incompleta / atrición: la edad de adquisición (EA), el tiempo de residencia en el entorno de la L2 (TR) y el nivel de contacto con la Lı (NC).

Investigamos, en particular, los posibles fenómenos de convergencia y/o transferencia invertida a los que nuestros sujetos de estudio, como usuarios adultos de una lengua, podrían ser susceptibles. Tomando como punto de partida hallazgos anteriores que validan la tesis de que los bilingües son vulnerables a fenómenos de transferencia bidireccionales tanto a nivel léxico (i. e., elección del tipo de verbos a utilizar en una descripción) como sintácticogramatical (i. e., estructuras de verbo más partículas y uso de frases preposicionales), nos preguntamos si dichos fenómenos afectarán la Lı de los bilingües aquí estudiados. A modo general, proponemos dos escenarios distintos:

1. La transferencia de los patrones tipológicos para la entrega de eventos de movimiento en la situación L2 > L1 es más frecuente e importante en aquellos hablantes que han tenido una edad temprana de inicio en la adquisición del sueco, mientras que aquellos individuos con una edad tardía de inicio en la L2 no presentan esta influencia en la misma medida.

2. Ambos grupos, los bilingües tempranos así como los bilingües tardíos, son susceptibles a la transferencia de los patrones del pensar para hablar del sueco L2.

Partiendo de las diferencias contrastivas entre el sueco y el español, ya descritas en el apartado 2, nos proponemos investigar la expresión formal de los siguientes aspectos: 1) la Manera (ya sea por medio de verbos principales o fuera de ellos en construcciones perifrásticas); 2) el Camino (i. e., entrega de partículas direccionales); y, finalmente, 3) la Base, ya sea aquella que aparece codificada por medio de complementos de destino/ punto final (PF) independientes, o bien por partículas satelitales unidas a un complemento ('sat. + PF').

Asimismo, esperamos encontrar patrones de transferencia similares a nivel intra-grupal, pero disímiles entre los bilingües tempranos y los tardíos, dando cuenta ello de que los fenómenos de transferencia y/o convergencia válidos para los bilingües tempranos reflejan un fenómeno distinto del que podría afectar a los bilingües tardíos. En cuanto a este último 
grupo, suponemos que una EA tardía, junto con un mayor contacto con la LI, representan variables que pueden minimizar los efectos de la transferencia de los patrones del "pensar para hablar".

\subsection{Método \\ 4.2.1. Participantes}

Los grupos que aquí se investigan están conformados de la siguiente manera:

a) Grupo de estudio (GE). Se trata de 31 sujetos bilingües de español LI/ sueco L2, cuyas edades al momento de participar en el estudio iban de los 20 a los 49 años (32,4 años en promedio). Un 68\% de los informantes, vale decir, 21 sujetos, son de origen chileno, mientras que el $32 \%$ restante procede de Perú (4), Colombia (2), Bolivia (1), México (1), Uruguay (1) y España (1). Todos los sujetos cuentan con educación media completa y la mayoría tiene incluso algún grado académico. La EA del sueco como L2 va del año hasta los 19 años y, en la mayoría de los casos, coincide con su edad de llegada a Suecia. Por otra parte, su TR en Suecia presenta un rango de $12-42$ años, con un promedio de 24,8 años. Respecto de sus habilidades en español, todos reconocieron por medio de un formulario tener algún grado de contacto diario con su LI; aún más, y en cuanto a su nivel de dominio en esta lengua, un test de juicio de gramaticalidad aplicado a este mismo grupo de informantes por Bylund (2009: 14) muestra que las diferencias obtenidas dentro de una comparación entre monolingües de español y el grupo de bilingües no arrojó diferencias significativas: $t(44)=-.987, p=.33$. En cuanto a su dominio del sueco, y de acuerdo a Abrahamsson y Hyltenstam (2009) en su estudio sobre 'logro máximo' en L2, un factor común a todos ellos es el que fueron seleccionados como grupo de estudio dado su alto nivel de proficiencia en sueco5.

b) Grupo de control sueco (GCS). Grupo compuesto por 11 hablantes nativos de sueco con conocimientos variados de inglés, alemán y francés aprendidos en la escuela. Ninguno de los sujetos declaró haber vivido en un país extranjero por una cantidad considerable de tiempo.

5 Los informantes, que previamente formaron parte del proyecto de Abrahamsson y Hyltenstam (2009), fueron seleccionados luego de haber sido juzgados como hablantes nativos de la L2 por un panel compuesto por hablantes nativos de sueco. El material presentado a este panel estuvo compuesto por conversaciones telefónicas en que los sujetos bilingües hablaban de la vida de la escritora sueca Astrid Lindgren, creadora de un personaje ampliamente conocido en el panorama literario de Suecia, la renombrada "Pippi calzas largas / medias largas", de tal manera que contaban con el conocimiento necesario como para hablar de ella sin lagunas de conocimiento. Dado que fueron seleccionados de acuerdo a su alto nivel de dominio en la $L 2$, el número de hablantes con EA más avanzada es más reducido que el número de hablantes con una EA más baja. 
Su edad promedio a la hora de participar en el test era de 33 años. Sus niveles de estudio son comparables a los de los grupos restantes: todos cuentan con estudios técnico-profesionales.

c) Grupo de control hispanófono (GCE). Grupo compuesto por 11 hablantes nativos de la variedad del español de Chile, país en que sus narraciones fueron recopiladas. No indicaron tener conocimientos de otras lenguas, excepto del inglés en un nivel básico. Al igual que los nativos de sueco, ninguno reconoció haber vivido en un país extranjero. Su edad promedio es de 28 años y su nivel de estudio es comparable, en general, al de los otros dos grupos.

\subsubsection{Materiales y procedimientos de recolección}

El corpus está compuesto por 53 narraciones orales de un cortometraje de once minutos y medio de duración Ilamado Quest (Thomas Stellmach, 1996). El mismo cortometraje, previamente utilizado por Von Stutterheim y colegas en una serie de estudios (por ej. en Von Stutterheim y Nüse (2003) y en Carroll, Von Stutterheim y Klein (2013), trata acerca de una figura de arena que atraviesa diversos mundos buscando agua. Cada mundo está representado por distintos materiales, como piedras y hojas de papel, y la figura de arena ha de usar una serie de artefactos con los que intentará romper la superficie de cada mundo para lograr hacer un agujero dentro de lo que él cree es una poza de agua.

Las narraciones producidas por el GE y el GCE fueron recopiladas por un hablante nativo del español de Chile y forman parte de una batería de pruebas lingüísticas que ha sido utilizada por, entre otros, Bylund (2009) y Abrahamsson y Hyltenstam (2009). Las narraciones correspondientes al GCS fueron compiladas posteriormente en sueco por el mismo administrador, quien cuenta con conocimientos avanzados en esta lengua. Las instrucciones dadas a los participantes, idénticas en todos los casos, consistieron en pedir a los sujetos que narraran el cortometraje luego de haber visto a la figura de arena pasar por cada mundo. Luego de cada episodio se les planteó la misma pregunta: “¿qué sucede en la película?”. Cada sujeto pudo contestar a esta pregunta haciendo uso del tiempo que estimara necesario.

Una vez recopilados los materiales, que fueron grabados en audio utilizando formato mp3, estos fueron transcritos con el software Transana por el administrador de los tests. Las transcripciones efectuadas en sueco, por su parte, fueron revisadas por un hablante nativo de esta lengua para descartar errores.

\section{Análisis y discusión}

A continuación se presentan los procedimientos de análisis y resultados obtenidos para cada uno de los aspectos formales descritos en el apartado 4.1. Estos aspectos son, seguidamente, 
discutidos en cuanto a las variables expuestas anteriormente: edad de adquisición de la L2 (EA), tiempo de residencia en el entorno de la L2 (TR), y nivel de contacto con la LI (NC).

\subsection{Entrega de la Manera por medio de verbos \\ 5.1.1. Procedimiento}

Como primer paso en el estudio del componente de Manera, se extrajo, por cada grupo de participantes, todos los verbos de movimiento. Dentro de este vasto conglomerado de verbos, no se consideró aquellos que hacen referencia a un estado o situación estática (como los verbos "brillar" o "divisar"), sino solo aquellos que expresan un movimiento, ya sea con desplazamiento (“correr”, "caer”, etc.) o sin él (“saltar", "resbalarse”, etc.). Una vez extraídos estos verbos, se procedió a crear una primera lista, que captura la frecuencia total de ocurrencias de verbos de movimiento; a partir de esta, se formaron dos sub-categorías: una de verbos de movimiento sin manera ("abrir", "caer”, "bajar”, etc.) y otra de verbos de manera-de-moverse ("arrastrase", "correr", etc.). Otras dos listas registran el número de tipos de verbos usados por cada participante: una para los verbos de movimiento sin manera y otra para los verbos-demanera de moverse ${ }^{6}$.

\subsubsection{Resultados}

Una comparación estadística de los resultados de los tres grupos (contando a los bilingües tempranos y tardíos, de momento, como un solo grupo) nos permite, como se describe a continuación, observar que los tres grupos de informantes (GE, GCE y GCS) operan de manera distinta. En cuanto al análisis del número total de ocurrencias de verbos de movimiento, la aplicación de un análisis de varianza (ANOVA) arroja un promedio de 41 verbos de movimiento para el GE (SD = 16.7), mientras que el GCE presenta un promedio de 51 (SD = 17.4) y el GCS un $56(S D=12.9)$. Estas diferencias resultan significativas, $F(2,50)=3.988, p=.025$. En otras palabras, los tres grupos se refieren a los eventos presentes en la película aportando un número disímil de verbos de movimiento (ver figura 1); diferencia que resulta significativa al aplicar un

6 Si bien el proceso de clasificación de los verbos de movimiento en 'verbos de movimiento con y sin manera’ es engorroso y debatible (Hijazo-Gascón y otros, 2013), hemos optado por clasificar los verbos de nuestro corpus basándonos en la clasificación de Morimoto (2001). Esta clasificación considera como verbo de manera a todo aquel verbo que conlleva una manera-de-moverse, ya sea con o sin desplazamiento. Por contraste, todo aquel verbo que no conlleva una forma específica de moverse ha sido catalogado como un verbo de movimiento sin manera (los verbos 'ir' y 'caer', por ejemplo, representan casos de verbos de movimiento sin manera). 
post-hoc (Tukey-Kramer) únicamente para la díada GE/GCS ( $p$ <.032). Por otro lado, el GE y el GCE no difieren significativamente, efecto que se repite para la comparación entre GCE y GCS.

\section{FIGURA 1}

Frecuencia promedio de ocurrencias de verbos de movimiento

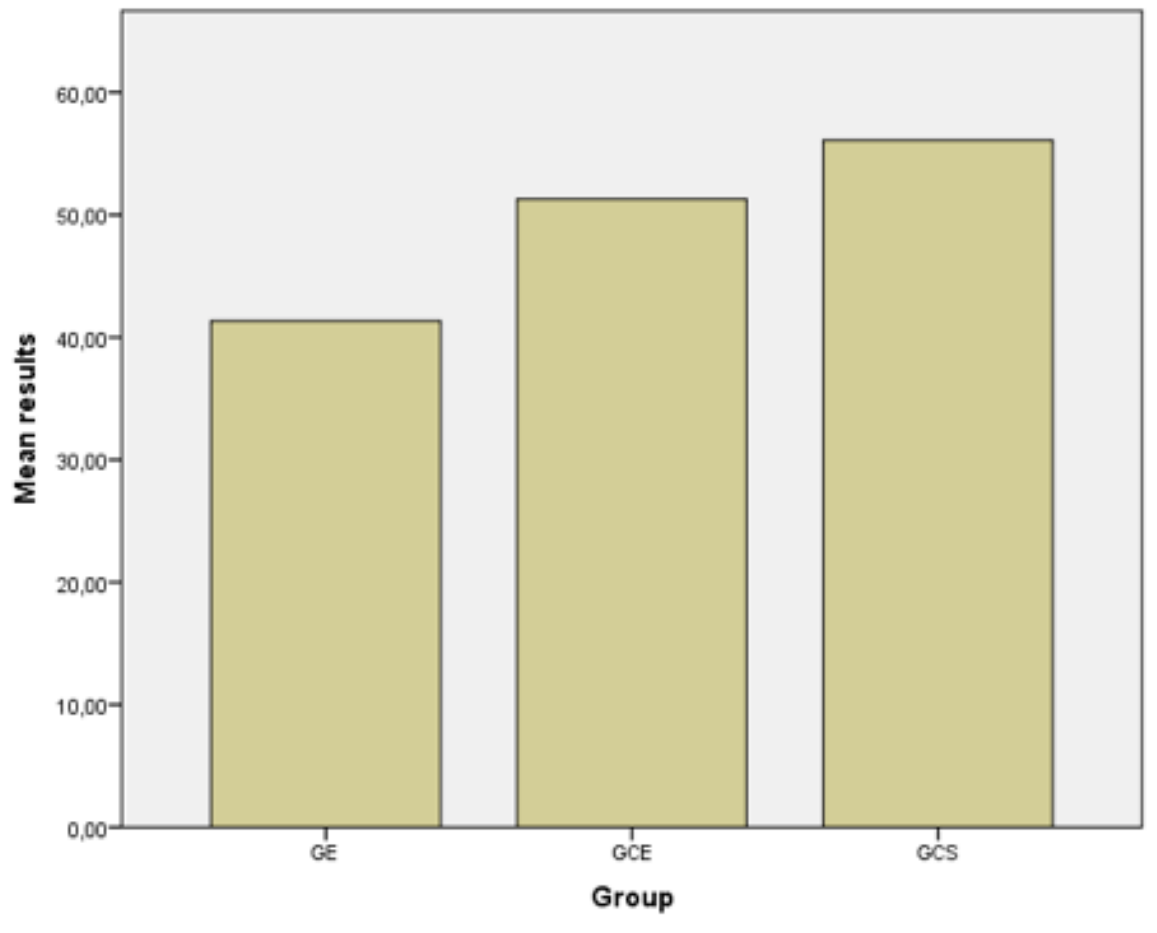

En la figura I se aprecia que los bilingües se acercan al número total de verbos usados por los hispanófonos, aunque no así al de los monolingües de sueco, quienes presentan un número significativamente mayor de verbos de movimiento, cuestión que se repite en estudios como los de Cadierno (2004), Cadierno y Ruiz (2006) y Donoso (2014). La pregunta que seguidamente salta a la vista es si los grupos diferirán en cuanto a la entrega de verbos de movimiento con y sin manera. Por esta razón se compararon tanto el porcentaje de uso de verbos sin manera con relación al número total de verbos de movimiento como el porcentaje de aparición de verbos-de-manera con relación al mismo grupo. La primera comparación arroja un porcentaje promedio para el GE de 65.7\% (SD = 9.1) de verbos sin manera; mientras que para el GCE es de $70 \%(S D=9.5)$ y de 51.2\% (SD = 8.1) para el GCS. La aplicación de una prueba ANOVA arroja

7 A lo largo de este estudio, el post-hoc utilizado en todos los casos corresponde al método TukeyKramer de comparaciones múltiples. 
al respecto una diferencia altamente significativa, $F(2,50)=14.077, p<.0001$. Seguidamente, un post-hoc nos permitió encontrar que tanto el GE como el GCS difieren significativamente entre sí ( $p<.001)$; aún más, la diferencia es asimismo altamente significativa entre los grupos de monolingües ( $p<.001)$, ver figura 2.

\section{FIGURA 2}

Porcentajes de uso de verbos de movimiento sin manera

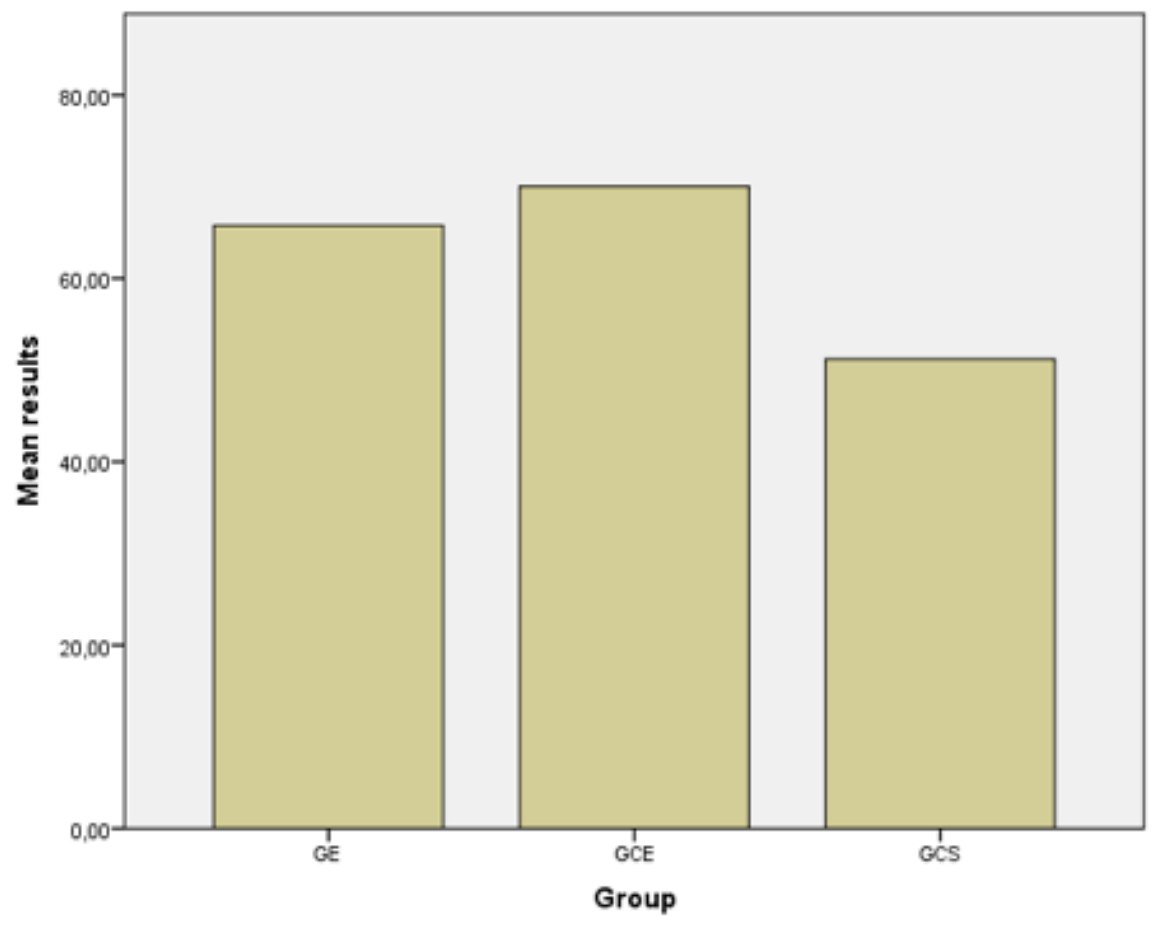

Estos hallazgos resultan comprensibles si tomamos en cuenta que el español es una lengua$V$, con una tendencia clara a lexicalizar la trayectoria en los verbos principales (aspecto que antes ha sido dado a conocer en la literatura en, p. ej. Sebastián y Slobin (1994) y Navarro y Nicoladis (2005), entre otros). Esto incidiría en la tendencia de los hispanófonos a usar verbos de movimiento carentes de manera incluso en aquellas situaciones en que, al menos en las lenguas-S, lo más usual es usar verbos-de-manera de moverse (Donoso, 2013). Los bilingües, por su parte, reflejan una tendencia a emular el patrón característico del español en cuanto a la lexicalización del Camino se trata; en otras palabras, no han perdido la capacidad de lexicalizar la trayectoria tal y como se hace en su LI.

En segundo lugar comparamos el porcentaje de uso de verbos-de-manera con el número total de verbos de movimiento. Aquí los resultados son de un 34.1\% en promedio para el GE $(S D=9.2)$, un $29.8 \%$ para el GCE (SD $=8.8)$ y un $48.7 \%$ para el GCS $(S D=8.1)$. Un ANOVA arroja 
en este caso, al igual que en el caso anterior, una diferencia altamente significativa, $F(2,50)$ $=14.253, p<.0001$. Luego, un post-hoc nos permitió establecer que estas diferencias son altamente significativas para la díada GE/ GCS (p<.001) y, además, altamente significativas entre los grupos de monolingües ( $p<.001)$, ver figura 3.

\section{FIGURA 3}

Porcentajes de uso de verbos-de-manera

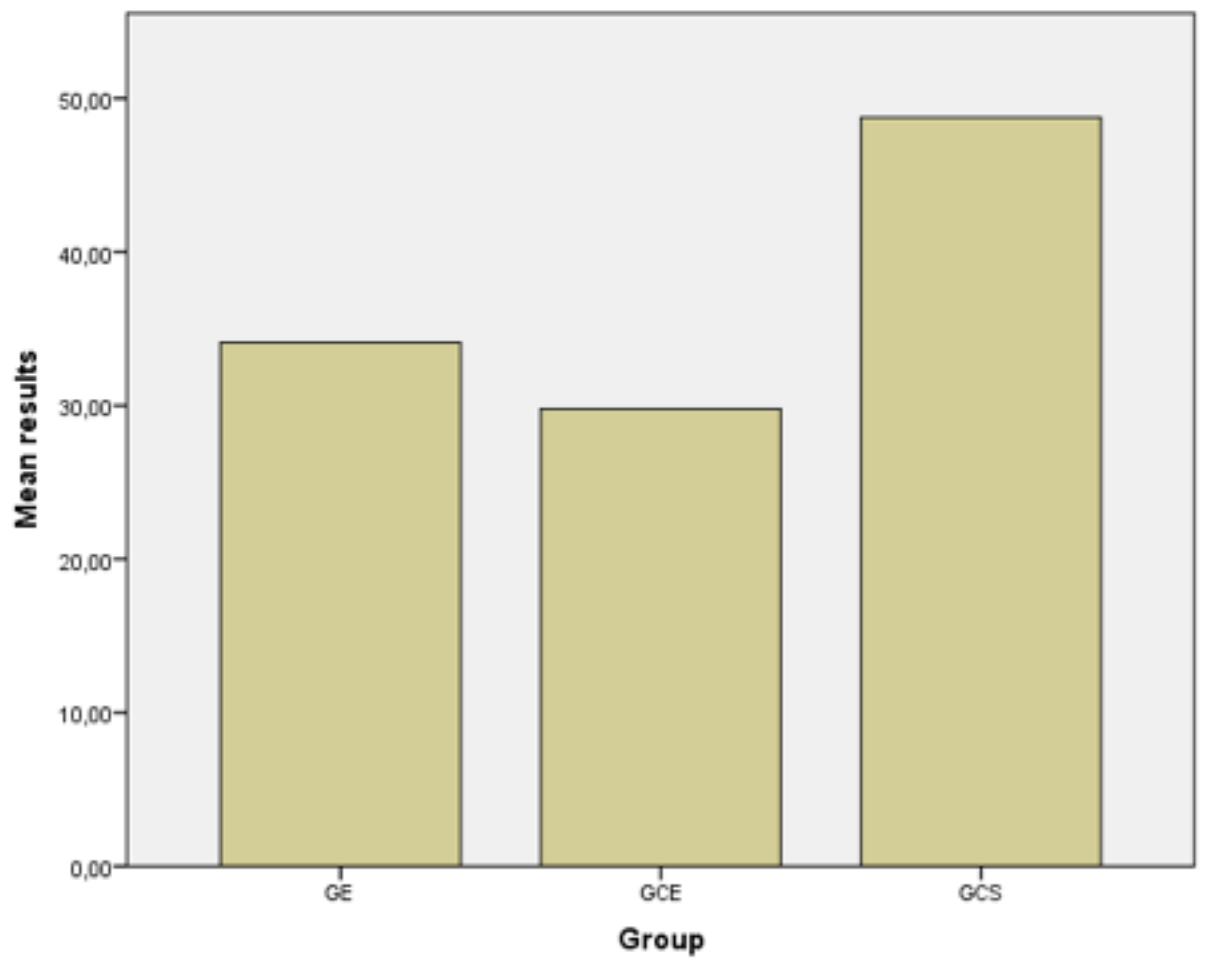

Como es posible apreciar, los monolingües de sueco utilizan verbos-de-manera de moverse con mayor frecuencia y superando, con creces, a los monolingües de español y a los bilingües. Por su parte, los bilingües se hallan ligeramente por encima de la media monolingüe en español, y, como vemos, muy por debajo del patrón sueco de lexicalización de la Manera; en este sentido, su baja frecuencia de uso de verbos-de-manera en español, y al menos en lo que a medidas cuantitativas se refiere, no se distancia mayormente de la frecuencia de los monolingües de esta lengua, lo que podría sin más llevarnos a la conclusión de que sus patrones preferidos de codificación de la Manera no han sido influidos mayormente por la L2 (cuestión análoga al estudio de la lexicalización del Camino en este mismo grupo). Ahora bien, si observamos en detalle los tipos de verbos utilizados (enfocándonos tanto en los verbos de movimiento sin manera como en los verbos-de-manera) podemos llegar, como se aprecia en los siguientes apartados, a unas conclusiones un tanto más matizadas. 
Por un lado, y tomando en cuenta solo los tipos de verbos de movimiento sin manera, obtuvimos los siguientes resultados: el GE presenta una media de 12 tipos (SD = 4.6); el GCE una media de $16(S D=4.2)$ y el GCS una media de 15. La aplicación de un ANOVA arroja diferencias significativas, $F(2,50)=5.091, p=.0097$. Luego, un post-hoc nos permitió establecer que las diferencias son significativas únicamente para el GE y el GCE ( $p<.05)$, resultado que nos permite entender que, frente a un evento de movimiento, los monolingües de español presentan un espectro de verbos sin manera significativamente más amplio que los bilingües, hallazgo natural si tomamos en cuenta que los nativos de español están habituados a lexicalizar el Camino de esta forma.

Por otra parte, y tomando en cuenta, esta vez, solo los tipos de verbos de manera-demoverse, nos encontramos con los siguientes resultados tras la aplicación de un ANOVA: el GE presenta una media de 9 tipos $(S D=3.9)$, el GCE de $10(S D=4.3)$ y el GCS de $17(S D=4.2)$. Estas diferencias son altamente significativas, con un valor $F(2,50)=14.534, p=.0001$. Esta vez, luego de aplicar un post-hoc, los grupos que difieren significativamente entre sí resultan ser el GE y el GCS ( $p<.001$ ) así como el GCE y el GCS ( $p<.01)$. Como vemos, en este caso el GE presenta un número de tipos verbales de manera cuantitativamente semejante a los monolingües de español; por otro lado, las diferencias que presentan los monolingües de sueco nos permiten concluir que estos recurren sistemáticamente a la lexicalización de la Manera característica de las lenguas-S, aspecto que no resulta totalmente permeable en la situación L2>L1. En otras palabras, y a nivel general en cuanto a la expresión de la Manera por medio de verbos concierne, los bilingües, a pesar de no contar con un repertorio muy alto de tipos de verbosde-manera en español, los eligen con mayor frecuencia que los hispanófonos (Figura 3) al referirse a este tipo de escenas.

\subsubsection{Verbos de movimiento con y sin manera a la luz de las variables EA, TR y NC}

Este tipo de análisis (que se repite más adelante en el estudio de la entrega de manera perifrástica, el Camino y la Base) se llevó a cabo introduciendo un "punto de inflexión" para cada una de las variables (EA, TR y NC), dividiéndose el GE en dos. En el caso de la EA, la inflexión se fijó a los once años ${ }^{8}$. De esta forma, se compararon con un t-test los resultados de quienes comenzaron a aprender sueco antes de los 12 años con los resultados de aquellos sujetos que comenzaron después de esta edad. Para el caso de la segunda

8 Esta suele ser la edad considerada como clave para muchos de los estudios llevados a cabo al alero de la hipótesis del período crítico (Lenneberg, 1967). 
variable en cuestión, el TR, el punto de inflexión se fijó en la media del grupo: 23 años de residencia; para el NC, el punto fue fijado en un $27 \%$ declarado de uso diario de la L1. Los resultados obtenidos al escrutar estas variables fueron comparados, al igual que en el caso de la EA, con la ayuda de un t-test. Ahora bien, y en lo que concierne a un análisis pormenorizado de la frecuencia de entrega de verbos de movimiento (con y sin manera), junto con un análisis de los tipos verbales a la luz de las variables EA, TR y NC, la aplicación de los t-tests correspondientes no arrojó ninguna diferencia estadística significativa, lo cual nos permite concluir, grosso modo, que los resultados no varían con relación a estas variables.

\subsection{Entrega de la Manera en construcciones perifrásticas y construcciones novedosas}

\subsubsection{Procedimiento}

Para evaluar este aspecto, cuantificamos la entrega de Manera en construcciones aledañas a los verbos principales, las que aquí denominamos 'construcciones perifrásticas' (algunos ejemplos tomados de esta lista son presentados más adelante en la Tabla 1). En esta clasificación se tomó en cuenta frases preposicionales que indican Manera, como, por ejemplo, en "cayó de cabeza" y también expresiones de Manera en gerundio (ej.: "y avanza sorteando obstáculos que atentan contra su vida”). Además, se hizo una recolección de usos novedosos (en este caso particular no considerados dentro del análisis estadístico).

\subsubsection{Resultados}

Los resultados, que aquí se obtuvieron a partir de una comparación entre la frecuencia de aparición de perífrasis con relación al número total de verbos de movimiento, son llamativos: el $\mathrm{GE}$, en promedio, proporciona una media de 12 perífrasis $(S D=7.9)$; el GCE $6(S D=6.4)$; y el GCS $9(S D=5.1)$. Si bien a simple vista estos resultados son bastante dispares entre sí, la aplicación de un ANOVA no arrojó una diferencia significativa, aunque el valor $p$ está muy cerca de ser inferior a 0.05: $F(2,50)=2.856, p=.067$. Sin embargo, y como podemos apreciar a partir de las medias de cada grupo, el GE presenta una mayor cantidad de perífrasis que el GCE, superando incluso al GCS (ver figura 4).

A continuación, en la tabla 1, presentamos algunos ejemplos de expresión de la Manera que difieren del español nativo en el GE; discutidos con mayor detenimiento, nos dan una idea de cómo se ve cualitativamente expresada la Manera en construcciones aledañas a los verbos de movimiento. 


\section{FIGURA 4}

Porcentajes de aparición manera perifrástica

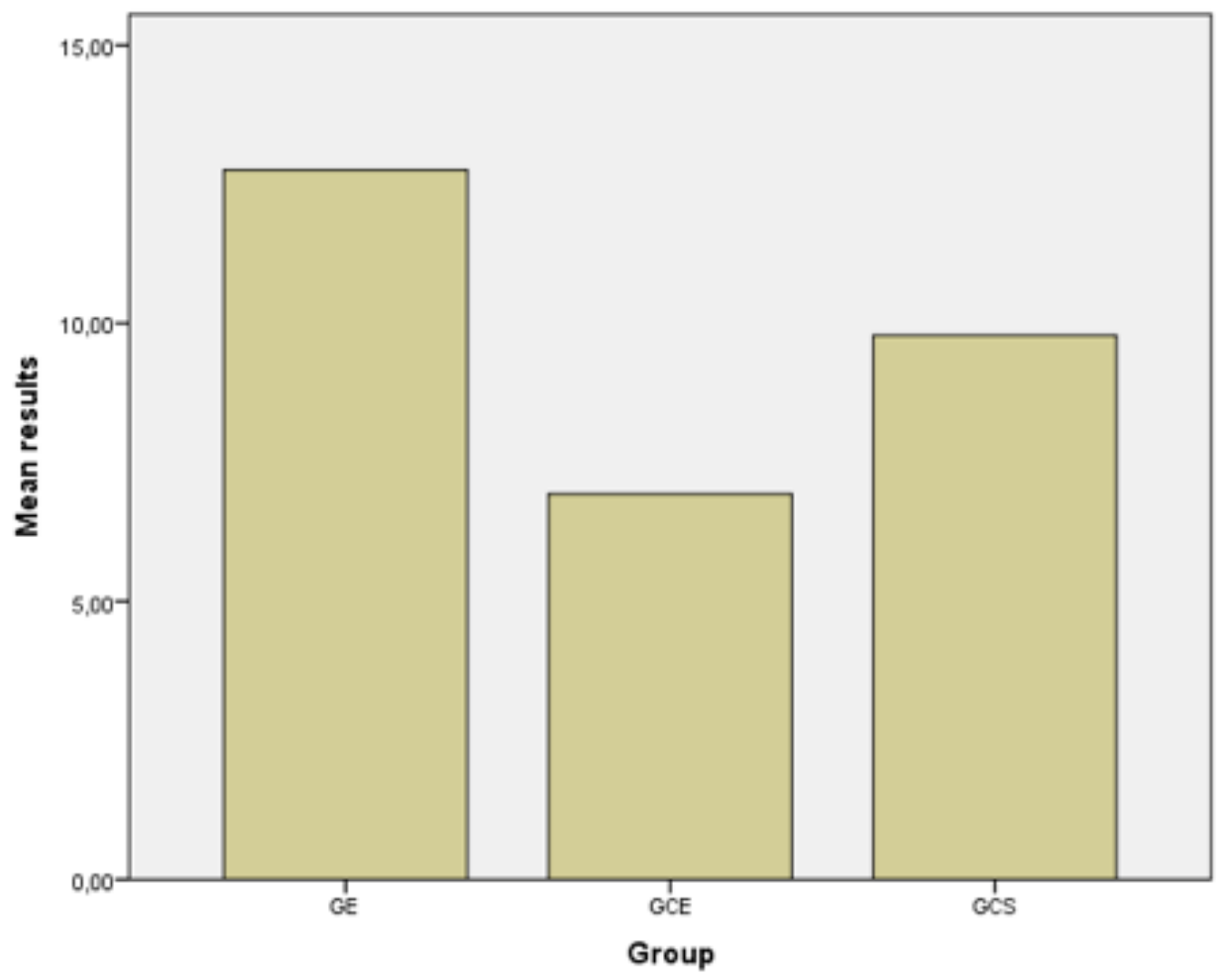

\section{TABLA 1}

Frecuencias gerundio 'no perifrástico' y forma -ing

\section{FRASES PREPOSICIONALES Y GERUNDIOS}

\section{(1) Y llegó de cabezas}

(2) Cayó de cabeza a otro nivel más bajo

(3) Y se paró en rodillas para esperar gotas de arriba

(4) Y cojeando se va a buscar el agua

(5) Se cae bajando

(6) Y el hombre se va cayendo por el hoyo hacia abajo

(7) Este ser humano cayó volando a otro mundo 
En la tabla 1 se aprecian algunos ejemplos de expresiones con gerundios y frases preposicionales (en negritas). Importante resulta destacar que su uso no está imposibilitado en español; sin embargo, en nuestras narraciones, su uso es poco recurrente en el GCE, aunque relativamente común en el GE. El ejemplo número (3), en el que debería decir "se paró DE rodillas para esperar gotas de arriba" en lugar de "*se paró EN rodillas...", aparece en la lista puesto que representa un error preposicional bastante común al interior del GE. En cuanto a los gerundios, algunos de los aquí expuestos aportan información 'extra' en muchos casos, como en (5) y (7). El ejemplo (6) constituye un caso frecuente en el GE de aglutinación de tres componentes centrales: Movimiento ('se va cayendo'), Camino ('hacia abajo') y Base ('por el hoyo'), en el que se ha optado por expresar todo de una vez, mediante una sola oración; sin duda, esta forma de expresar un evento en español resulta, además de redundante, poco frecuente en nuestros datos. Por otro lado, y en cuanto a los casos que representan usos novedosos y/o giros idiosincrásicos, algunas oraciones contienen verbos que no existen en el español nativo, pero que resultan efectivos en la comunicación. Ejemplo de ello serían las oraciones "y de repente como que se inunde" o la oración "y su corazón empezó a pulpir más y más fuerte". Estos casos, al parecer, representan desplazamientos léxicos en que dos vocablos distintos convergen en una creación original: 'hundirse + inundar' > inunda; 'palpitar + latir' > pulpir. Por otra parte, casos como el de "el hombre trató de excavarse” o el de la oración "y fue destrozado a arena” constituyen calcos de expresiones resultativas suecas: "el hombre trató de excavarse" > mannen försökte gräva sig ut ('el hombre trató de excavarse a sí mismo hacia afuera’); "y fue destrozado a arena > han smulades till sand ('él fue destrozado y reducido a arena').

\subsubsection{Manera perifrástica a la luz de las variables EA, TR y NC}

En este caso, los hallazgos resultaron significativos únicamente para la variable TR $(t=2.189$, $d f=29, p=.0368)$. El promedio obtenido para los bilingües con $T R<23$ años fue de un $9.2 \%$ de casos de manera perifrástica $(S D=5.7)$, mientras que los bilingües con TR>23 años proporcionaron, en promedio, un 15.2\% (SD=8.5). En otras palabras, quienes Ilevan más tiempo en Suecia son más susceptibles a ser influidos por el patrón de entrega de Manera proveniente de la L2 favoreciendo el uso de oraciones que contienen el componente de Manera. Aparentemente, esta estrategia de uso común en el GE refleja un caso de transferencia conceptual: el alto nivel de uso de distintos tipos de verbo de manera en la L2 impactaría en el GE aumentando su necesidad de codificar la Manera, incluso fuera de los verbos principales. Estos resultados están en línea con los hallazgos de Donoso y Bylund (2015), quienes encontraron algo similar en sus sujetos estudiados, aunque en la situación L1>L2 (un grupo de hablantes de español L2 con sueco como L1 que al momento de ser investigado presentaba un alto promedio de estadía en el entorno de la L2). 


\subsection{Uso de partículas direccionales}

\subsubsection{Procedimiento}

En cuanto a las partículas satelitales, se cuantificó el número total de partículas usadas junto a un verbo de movimiento por cada narración. Estas partículas expresan necesariamente una dirección (ut, 'hacia afuera'; in, 'hacia adentro'; upp, 'hacia arriba'; etc.). Casos tomados del grupo de bilingües, en español Lı, que ejemplifican estos usos son:

(4) y siguió para abajo la arena

(5) y tiene que trepar para abajo otra vez

(6) y entonces lo subieron arriba de las rocas

En algunos casos, las partículas direccionales constituyen adverbios deícticos (como ahí o alli). Ejemplos serían:

(7) y se fue para allá

(8) alcanzó a tirarse por ahí antes de que la máquina lo alcanzara

(9) y fue allí donde caía el agua

\subsubsection{Resultados}

El análisis de varianza efectuado con un ANOVA nos permitió hallar un promedio de 3.5 partículas para el GE $(S D=3)$; 0.45 para el GCE $(S D=0.8)$; y 25 para el GCS $(S D=6.6)$. Este hallazgo resulta significativo: $F(2,50)=164.84, p=.0001$. Luego, la aplicación de un post-hoc nos permite apreciar que las diferencias son significativas para el GE y el GCS (p<.001) y para la díada GCE y GCS (p<.001), aunque no para el GE y el GCE (ver Figura 5).

En otras palabras, los bilingües no satelizan significativamente más que los monolingües de español, aunque sí con una frecuencia relativamente mayor, cuestión que analizamos a la luz de las variables EA, TR y NC a continuación.

\subsubsection{Satelización a la luz de las variables EA, TR y NC}

En este caso, la aplicación de los t-tests correspondientes arroja resultados estadísticamente significativos única y exclusivamente para la variable $E A(t=3.124, d f=29, p=.0040)$. El promedio para los bilingües con una $E A<12$ es de 4.6 satélites $(S D=2.9)$, mientras que para los que 


\section{FIGURA 5}

Frecuencia de entrega de partículas satelitales

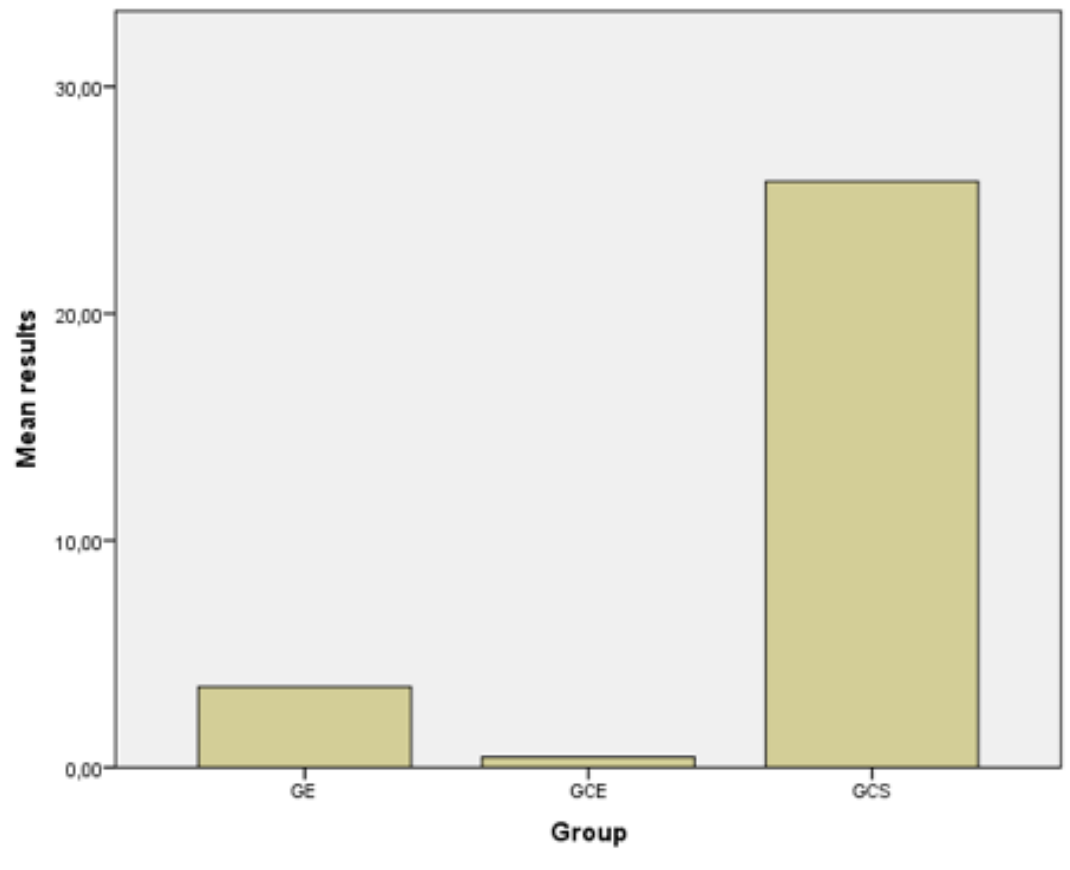

tienen una $E A>12$ es de 1.5 satélites $(S D=2)$. En otras palabras, en quienes han llegado a Suecia más temprano, y, por ende, tienen una baja EA9 , el patrón sueco de expresión del Camino parece haberse vuelto más prominente: la tendencia general en estos individuos es a entregar la dirección por medio de un satélite, a pesar de que el Camino aparece lexicalizado en español al interior del verbo principal. Estos hallazgos son discutidos en mayor detalle en el apartado 6.

\subsection{Complementación: entrega de puntos finales \\ 5.4.1. Procedimientos}

En cuanto al componente de Base, se cuantificó solo aquellos complementos circunstanciales que indican un destino o punto final (PF). Tal sería el caso de, por ejemplo, el complemento "a un tipo de fábrica” en la oración siguiente:

9 Nótese que las variables edad de adquisición de la L2 y tiempo de residencia en el entorno de la L2 no presentan una correlación estadísticamente significativa. En otras palabras, quienes Ilegaron a Suecia a una edad más temprana no necesariamente son quienes Ilevan residiendo en el país por una mayor cantidad de tiempo. 
(10) y cae a un tipo de fábrica

Asimismo, se ha tomado en cuenta la aparición de puntos finales unidos a un satélite (i. e., la frecuencia de aparición de la estructura 'sat. + PF'). Ejemplos:

(11) y se cayó abajo a un hoyo

(12) y siguió pa' abajo al lado de la botella de agua

\subsubsection{Resultados}

En cuanto a la entrega de PF, el promedio para el GE es de 9 PF (SD = 3); 13 para el GCE $(S D=5)$; $y$ 14 para el GCS $(S D=5)$. El resultado de una ANOVA arroja una diferencia significativa de $F(2,50)$ = 9.302. De acuerdo a un post-hoc, los grupos que difieren son el GE y el GCE ( $p=.05)$, y la díada GE y GCS ( $p=.001)$. Los monolingües no difieren significativamente entre sí. En otras palabras, los bilingües entregan un número significativamente menor de PF si los comparamos con los monolingües de ambas lenguas (ver figura 6).

Por otro lado, la entrega de la estructura 'satélite + PF' resulta, asimismo, ser significativa. En promedio, el GE presenta esta estructura 1.3 veces $(S D=1.3)$; el GCE $0.2(S D=0.4)$; y el

\section{FIGURA 6}

Frecuencia de entrega de puntos finales

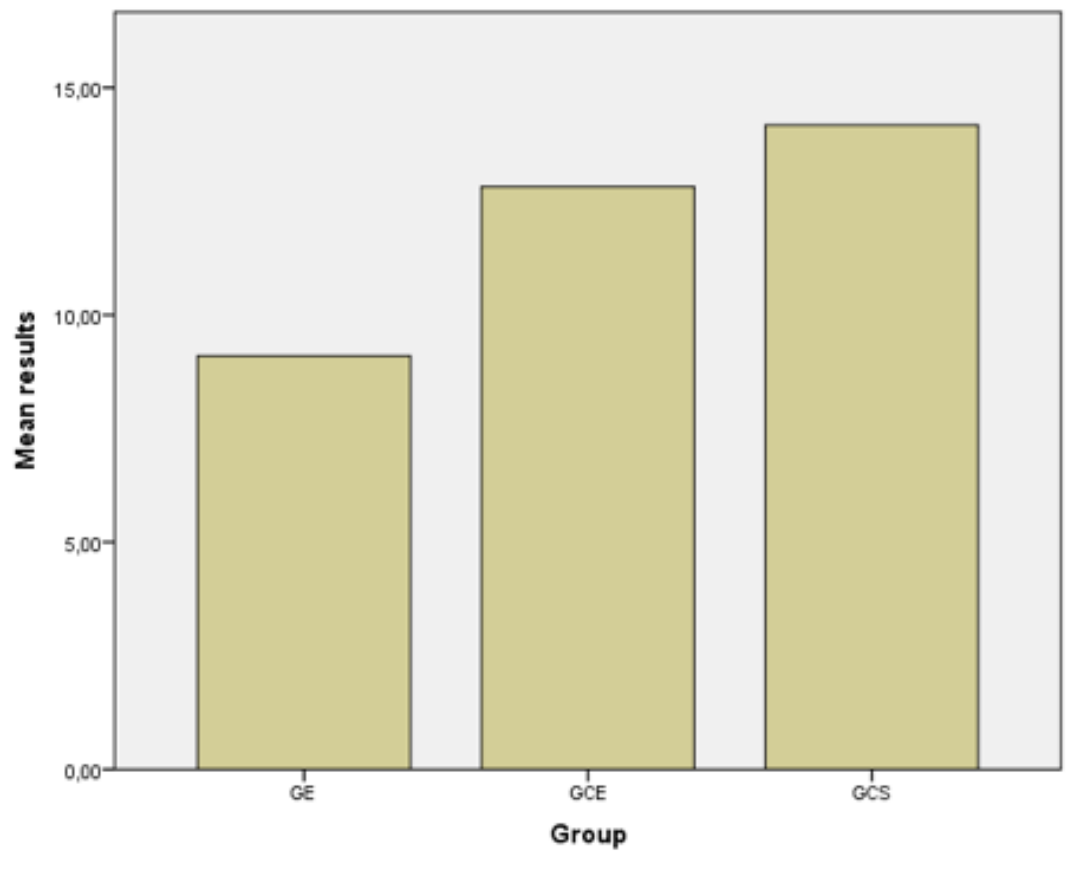


GCS $8.6(S D=3.1)$. Un ANOVA arroja una diferencia significativa de $F(2,50)=80.103, p=.0001$. El post-hoc nos permite ver que los grupos que difieren entre sí son esta vez las díadas GE/ GCS (p<.001) y GCE/ GCS (p<.001). En otras palabras, los bilingües presentan la estructura 'satélite + PF' con una frecuencia significativamente menor que los monolingües de sueco. Los grupos de monolingües difieren, a su vez, significativamente entre sí. Esto quiere decir que, a simple vista, los bilingües consiguen adaptarse al patrón hispanófono de codificación del Camino y la Base (ver figura 7).

\section{FIGURA 7}

Frecuencia de entrega de 'satélites + puntos finales'

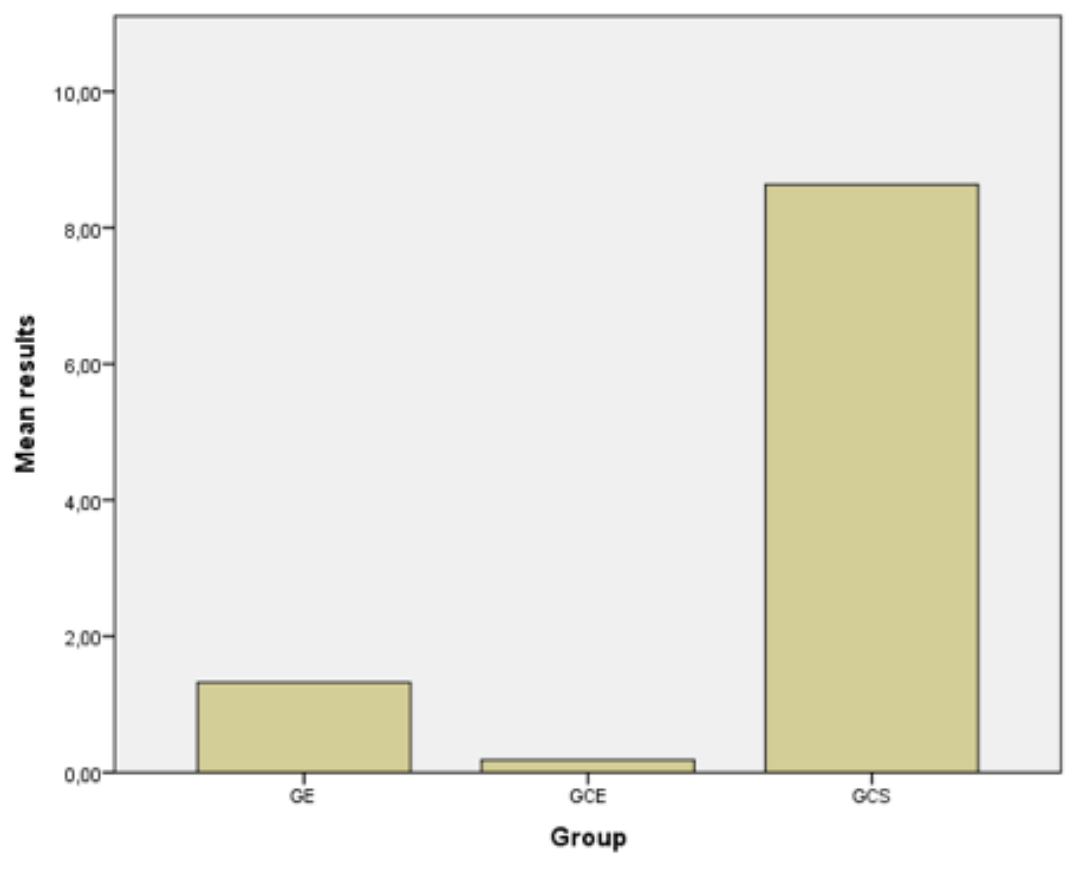

A pesar de los resultados recién expuestos, y ante el hallazgo de que esta emulación del patrón monolingüe español no es el del todo idéntico, analizamos nuestros hallazgos a la luz de las variables EA, TR y NC en el apartado siguiente.

\subsubsection{Entrega de puntos finales a la luz de las variables EA, TR y NC}

En este caso, los resultados de los t-tests no arrojaron diferencias estadísticamente significativas para ninguna de las variables con relación a la entrega de puntos finales, pero sí en cuanto a la entrega de la estructura 'satélite + PF' y la EA de la L2 $(t=2.385, d f=29, p=.0238)$. En promedio, los bilingües con una EA<12 años presentan 1.8 estructuras de este tipo $(S D=1.5)$, 
mientras que los que tienen una EA>12 exhiben $0.5 \operatorname{casos}(S D=0.9)$. En otras palabras, los bilingües con una edad temprana de adquisición son más susceptibles a utilizar la estructura 'satélite + PF' que aquellos que han llegado más tarde.

\section{Discusión y conclusiones generales}

Este estudio, cuyo objetivo principal ha sido el de caracterizar las narraciones de un grupo de bilingües de español/ sueco en cuanto a cómo proporcionan la información de Camino, Base y Manera de movimiento, tiene como premisa que este tipo de información diferirá en el grupo observado dependiendo de tres variables centrales: la edad de adquisición (EA) de la $L 2$, el tiempo de residencia (TR) en el entorno de la $L 2$ y el nivel de contacto (NC) que presentan con la Lı. Nuestra hipótesis es que los patrones de conceptualización tenderán a diferenciarse de la norma nativa de la L1, particularmente en aquellos casos en que los bilingües han adquirido una L2 antes de la pubertad. Al respecto, se predicen dos escenarios distintos, dependiendo de si la hipótesis planteada anteriormente es válida o no: por un lado, la transferencia de los patrones tipológicos de la L2 a la Lı será más frecuentes en aquellos sujetos con una edad temprana de adquisición de la L2, mientras que aquellos individuos que han adquirido la L2 luego de la pubertad no presentarán esta influencia en la misma medida; por otro, se plantea que ambos grupos serán susceptibles a la transferencia de los patrones del "pensar para hablar" del sueco como L2.

Los resultados se obtuvieron a partir del análisis de los siguientes aspectos formales: 1) la Manera, aspecto que fue escrutado por medio de un análisis pormenorizado de los tipos/ ocurrencias verbales, y a través de un análisis de la entrega de la Manera en construcciones perifrásticas; el Camino, ámbito que se estudió en cuanto a la entrega de partículas satelitales; y la Base, aspecto de la expresión del movimiento que se estudió en cuanto a la entrega de complementos de destino o punto final, y la entrega de satélites más este mismo tipo de complementos.

Nuestros hallazgos apuntan en una dirección, en general, bastante clara: tanto los bilingües tempranos como los tardíos son susceptibles al influjo de los patrones del "pensar para hablar" de la L2, aunque no en la misma medida y de forma divergente. Sucintamente, hemos podido constatar que los mayores efectos se producen en cuanto a la entrega de manera perifrástica, la entrega de satélites, y la expresión del Camino y la Base por medio de satélites más complementos de destino. Estos resultados varían entre los grupos de bilingües solamente en lo que se refiere a las variables EA y TR; el impacto de la variable NC pudo ser descartado.

En cuanto a la alta entrega de manera perifrástica, aspecto que se evidencia de forma estadísticamente significativa en los sujetos con mayor tiempo de residencia en Suecia, los hallazgos pueden ser explicados si echamos mano a la hipótesis de la transferencia concep- 
tual: aparentemente estos sujetos, al igual que los sujetos estudiados por Hohenstein y otros (2006), se han visto habituados a la alta frecuencia con que este componente está presente en el input. Su tiempo de estadía en el entorno de la L2, superior a los 23 años, les habría vuelto más sensibles a la Manera en que distintos eventos ocurren, reflejándose ello en la entrega frecuente, creativa, y en muchos casos poco idiosincrásica, de perífrasis de Manera. Recordemos que, por otro lado, el grupo de bilingües, visto como un solo grupo y comparado con los monolingües de español, presenta una predilección por el uso de verbos de manera por sobre otros tipos de verbos en aquellos casos en que este componente puede ser expresado por medio de un verbo. Notoriamente, su espectro de tipos verbales de manera no es superior al de los hispanófonos; sin embargo, el uso de verbos-de-manera de moverse es mayor en los bilingües que en los monolingües de español.

Por otro lado, y en cuanto a la entrega de satélites se refiere, los resultados apuntan a que los bilingües con una EA temprana presentan una tendencia a utilizar estos recursos para expresar el Camino con una mayor frecuencia que los bilingües tardíos. El impacto de la EA es uno que ha podido constatarse en una serie de estudios anteriores, particularmente en los de Bylund (2009) y Bylund y Jarvis (2011). Según estos autores, la habituación a una forma específica de hacer referencia al movimiento tiene un impacto ineludible en aquellos individuos con una baja EA, efecto que se ha encontrado, además, en los gestos que acompañan a la entrega de eventos de movimiento en sujetos bilingües (Bylund y Jarvis, 2011: 51). De esta forma, y tomando en cuenta que los bilingües tempranos habrían alcanzado a adquirir la L2 antes de la pubertad, no es difícil imaginar que sus patrones de expresión del Camino se verían profundamente traspasados por los patrones característicos del sueco. De acuerdo a estudios como los de Bowerman y otros (1996) y los de Choi y Bowerman (1991), los patrones específicos de codificación del movimiento de una Lı tendrían un impacto en la conceptualización de los hablantes de una lengua determinada desde una edad muy temprana (desde antes de los dos años de edad), cuestión que podría verse reflejada en la entrega de abundantes satélites direccionales en los bilingües tempranos aquí investigados. Por otro lado, y dado que la entrega de satélites, si bien redundante, no está vetada en español, comprensible resulta entonces que nuestros sujetos bilingües con una menor EA echen mano a estos recursos. Los bilingües tardíos, por su parte, no habrían perdido la sensibilidad frente al hecho de que muchos de los verbos de movimiento en español ya contienen el componente de Camino, razón por la que evitarían su uso. Interesante resultaría, en este caso, estudiar estos mismos grupos de individuos, pero analizando, esta vez, su producción oral en sueco.

Finalmente, y en cuanto a la entrega de satélites más puntos finales concierne, nuestros resultados apuntan a que este tipo de estructura se da con mayor frecuencia, nuevamente, en los bilingües tempranos, aspecto que difiere de lo que ocurre con los bilingües con una alta EA del sueco. Este esquema 'témporo-espacial', característico en general de las lenguas escandinavas (Pedersen, 2009), sería transferido de forma directa desde la L2 a la L1, puesto 
que nada en el español les diría, al igual que en el caso de los satélites, que su mención puede resultar atípica y redundante. Siguiendo a Bylund y Jarvis (2011), determinadas estructuras de frecuencia recurrente presentes en el input al que los individuos están habituados (en este caso dado su temprano inicio en la adquisición del sueco), pueden haberse vuelto rutinarias en su forma de codificar eventos de movimiento.

En resumen, y si bien hay puntos que divergen, resulta evidente que los bilingües tempranos y tardíos operan con dos sistemas lingüísticos al momento de elegir y organizar la información de movimiento en su LI, eligiendo aquello a lo que se han vuelto más sensibles dado su uso significativo de dos lenguas (recordemos que como grupo se trata de sujetos con un alto dominio del sueco como L2); luego, organizan su discurso en base a aquello que funciona en el contexto de la legua en uso, aunque no necesariamente resulte totalmente típico. Sus narraciones contendrían, por tanto, un esquema mixto de recursos lingüísticos, algunos más cercanos a la Lı (particularmente en el caso de los bilingües tardíos); otros basados en los patrones esquemáticos de la L2 (especialmente en los bilingües con una EA temprana). En consecuencia, argumentamos que estos dos grupos de individuos han de considerarse como sujetos susceptibles a distintos fenómenos de transferencia y/o convergencia.

\section{Bibliografía citada}

Abrahamson, Niclas y Kenneth Hyltenstam, 2009: "The robustness of aptitude effects in near-native second language acquisition", Studies in Second Language Acquisition 30, 481-509.

Beavers, John, Beth Levin y Shiao Wei Tham, 2010: "The typology of motion expressions revisited", Journal of linguistics 46, 331-377.

Bowerman, Melissa, Lourdes De León y Soonja Chol, 1995: "Verbs, particles, and spatial semantics: Learning to talk about spatial actions in typologically different languages" en Eve V. CLark (ed.): Proceedings of the Twenty-seventh Annual Child Language Research Forum, Stanford, CA: Center for the Study of Language and Information, 101-110.

Brown, Amanda y Marianne Gullberg, 2011: "Bidirectional cross-linguistic influence in event conceptualization? Expressions of Path among Japanese learners of English", Bilingualism: Language and Cognition 14 (1), 79-94.

ByLund, Emanuel, 2009: "Effects of age of L2 acquisition on L1 event conceptualization patterns", Bilingualism: Language and Cognition 12, 305-322.

ByLund, Emanuel, 2011: "Segmentation and temporal structuring of events in early SpanishSwedish bilinguals", International Journal of Bilingualism 15, 86-54. 
Bylund, Emanuel y Scott Jarvis, 2011: "L2 effects on L1 event conceptualization”, Bilingualism: Language and Cognition 14, 47-59.

Cadierno, Teresa, 2004: "Expressing motion events in a second language: A cognitive typological approach" en Michel Achard y Susanne Neimeler (eds.): Cognitive linguistics, second language acquisition, and foreign language teaching, SOLA Studies on Language Acquisition, Berlin: Mouton de Gruyter, 13-49.

Cadierno, Teresa, 2008: "Learning to talk about motion in a foreign language" en Peter Robinson y Nick Elus (eds.): Handbook of cognitive linguistics and second language acquisition, New York / London: Routledge, 239-275.

Cadierno, Teresa, 2010: "Motion in Danish as a second language: Does the learner's L1 make a difference?” en ZhaoHong Han y Teresa CADIERno (eds.): Linguistic relativity in second language acquisition: Thinking for speaking, Bristol: Multilingual Matters, 1-33.

Cadierno, Teresa y Lucas Ruiz, 2006: "Motion events in Spanish L2 acquisition” en Annual Review of Cognitive Linguistics 4, 183-216.

Cadierno, Teresa y Peter Robinson, 2009: "Language typology, task complexity and the development of L2 lexicalization patters for describing motion events", Annual Review of Cognitive Linguistics 7, 245-276.

Carroll, Mary, Christiane Von Stutterheim y Ralph Nüse, 2004: "The language and thought debate: A psycholinguistic approach" en C. Habel y T. Pechmann (eds.): Multidisciplinary approaches to language production, Berlin: Mouton de Gruyter, 183-218.

Carroll, Mary, Christiane von Stutterheim y Wolfgang Klein, 2003: "Two ways of construing complex temporal structures” en Friedrich Lenz (ed.): Deictic conceptualisation of space, time and person, Amsterdam: Benjamins, 97-134.

Chol, Soonja y Melissa Bowerman, 1991: "Learning to express motion events in English and Korean: The influence of language-specific lexicalization patterns”, Cognition 41, 83-121.

Cook, Vivian James (ed.), 2003: Effects of the second language on the first, Buffalo: Multilingual Matters.

DEKEYSER, Robert, 2000: "The robustness of critical periods effects in second language acquisition", Studies in Second Language Acquisition 22, 499-533.

Donoso, Alejandra, 2013: "Camino, Base y Manera en español y sueco", Revue Romane 48(1), 1-31. 
Donoso, Alejandra, 2014: "The impact of Swedish Lion the expression of path and manner in Spanish L2", Fagbokforlaget Nordand -Nordisk tidsskrift for andrespråksforskning 9(2), 47-72.

Donoso, Alejandra y Emanuel ByLund, 2015: "The Construal of Goal-Oriented Motion Events by Swedish Speakers of L2 Spanish: Encoding of motion endpoints and Manner of motion" en Judy Tiffany y Silvia Perpiñan (eds.): The Acquisition of Spanish in Understudied Language Pairings, John Benjamins, 233-254.

Flecken, Monique, Christiane Von Stutterheim y Mary Carroll, 2014: "Grammatical aspect influences motion event perception: evidence from a cross-linguistic, non-verbal recognition task", Language and Cognition 6(1), 45-78.

HAKUtA, K. y Daniel D'AndREA, 1992: "Some properties of bilingual maintenance and loss in Mexican Background high-school students", Applied Linguistics 13(2), 72-99.

Hendriks, Henriëtte y Hickmann MaYA, 2011: "Space in second language acquisition" en Vivian Cook y Benedetta Bassett। (eds.): Language and Bilingual Cognition, Hove, UK: Psychology Press, 315-339.

Hijazo-GasCón, Alberto, 2011: La expresión de eventos de movimiento y su adquisición en segundas lenguas. Tesis doctoral, Universidad de Zaragoza en España.

Hijazo-Gascón, Alberto, Iraide Ibarketxe-Antuñano y Julia Guelbenzu-Espada, 2013: "Clasificando los verbos de movimiento. ¿Qué piensan los hablantes?” en J. F. VAL, J. L. MendiviL, M. C. Horno, I. Ibarretxe, A. Hijazo, J. Simón e I. Solano (eds.): Actas del $10^{\circ}$ Congreso Internacional de Lingüística General / Proceedings of the 1oth International Conference on General Linguistics, Zaragoza: Prensas Universitarias de Zaragoza.

Hohenstein, Jill, Ann EIsenberg y Letitia Naigles, 2006: "Is he floating across or crossing afloat? Cross-influence of L1 and L2 in Spanish-English bilingual adults", Bilingualism: Language and Cognition 9, 249-261.

IBAÑez-Cerda, Sergio, 2002: "El clítico se en los verbos de movimiento intransitivos del español. Un análisis en términos de estructura temática”, Nueva Revista de Filología Hispánica 1, 169180.

Ibarretxe-Antuñano, Iraide, 2009: "Path Salience in Motion Events" en Jiansheng Guo, Elena Lieven, Nancy Budwig, Susan Ervin-Tripp, Kei Nakamura y Seyda Özçalşkan (eds.): Crosslinguistic Approaches to the Psychology of Language: Research in the Tradition of Dan Isaac Slobin, New York: Psychology Press, 403-414. 
Jarvis, Scott y Aneta Pavlenko, 2008: Crosslinguistic influence in language and cognition. New York: Routledge.

Jovanovic, Jelena y Aida Martinovic-Zic, 2004: "Why manner matters. Contrasting English and Serbo-Croatian typology in motion description" en Carol Lynn Moder y Aida Marti-Novic-Zic (eds.): Discourse Across Languages and Cultures, Philadelphia: John Benjamins Publishing Company, 211-226.

KöPkE, Barbara y Monika Schmid, 2004: “Language attrition: The next phase” en Monika Schmid, Barbara KöPke, Merel KeIJZER y Lina WeILEMAR (eds.): First language attrition: Interdisciplinary perspectives on methodological issues, Amsterdam: John Benjamins, 1-43.

Larrañaga, Pilar, Jeanine Treffers-Daller, Françoise Tidball y Mari-Carmen Ortega, 2012: "LI transfer in the acquisition of manner and path in Spanish by native speakers of English", International Journal of Bilingualism 16(1), 117-138.

LenneberG, Eric, 1967: Biological foundations of language, New York: Wiley.

Montrul, Silvina, Rebecca Foote y Silvia Perpiñán, 2008: "Gender agreement in adult second language learners and Spanish heritage speakers: The effects of age and context of acquisition", Language Learning 58(3), 503-553.

Morimoto, Yuko, 2001: Los verbos de movimiento, Madrid: Visor Libros.

Naigles, Letitia y Paula TerRAzAs, 1998: "Motion-verb generalizations in English and Spanish: Influences of language and syntax", Psychological Science 9(5), 363-369.

NavarRo, Samuel y Elena Nicoladis, 2005: “Describing motion events in adult L2 narratives" en David Edington (ed.): Selected Proceedings of the $6^{\text {th }}$ Conference on the Acquisition of Spanish and Portuguese as First and Second Languages, Somerville, MA: Cascadilla Proceedings Project, 102-107.

Pavlenko, Aneta y Scott Jarvis, 2000: "Bidirectional transfer”, Applied Linguistics 23, 190-214.

Pedersen, Johan, 2009: "Lexical and constructional organization of argument structure: A contrastive analysis" en J. Zlatev, M. Johansson Falck, C. Lundmark y M. Andrén (eds.): Studies in Language and Cognition, Cambridge Scholars Publishing, Newcastle-upon-Tyne, 230-245.

Schmiedtova, Barbara y Monique Flecken, 2008: "The role of aspectual distinctions in event encoding: Implications for second language acquisition" en S. de KNOP y T. de Rycker (eds.): Cognition Approaches to Pedagogical Grammar, Berlin: De Gruyter, 357-384. 
Schmiedtova, Barbara, Christiane Von Stutterheim y Mary Carroll, 2011: "Language-specific patterns in event construal of advanced second language speakers" en Aneta PAvLEnko (ed.): Thinking and Speaking in Two Languages: Clevedon: Multilingual Matters, 112-141.

Sebastián, Eugenia y Dan Slobin, 1994: "Development of linguistic forms: Spanish" en Ruth A. Berman y Dan Slobin (eds.): Relating events in narrative: A crosslinguistic developmental study, Hillsdale, NJ: Lawrence Erlbaum, 239-284.

Silva-Corvalán, Carmen, 1994: Language contact and change. Spanish in Los Angeles, Oxford: Claredon Press.

SנöstRöm, Sören, 1990: Spatial Relations. Towards a theory of spatial verbs, prepositions and pronominal adverbs in Swedish. Tesis doctoral, Institutionen för filosofi, lingvistik och vetenskapsteori. Göteborgs universitet.

SLobIN, Dan y Aurora Bocaz, 1988: "Learning to talk about movement through time and space: The development of narrative abilities in Spanish and English", Lenguas Modernas 15, 5-24.

SLobIn, Dan, 1991: "Learning to think for speaking: Native language, cognition, and rhetorical style", Pragmatics 1, 7-25.

SLobin, Dan, 1993: "Adult language acquisition: A view from child language” en Clive Perdue (ed.): Adult language acquisition: Cross-linguistic perspectives, Cambridge: Cambridge University Press, 239-252.

SLobIn, Dan, 1996: "From 'thought and language' to 'thinking for speaking'” en John Gumperz y Stephen Levinson (eds.): Rethinking linguistic relativity, Cambridge: Cambridge University Press, 70-96.

Slobin, Dan, 1997: “Mind, code, and text” en Joan L. Bybee, John Haiman y Sandra A. Thompson (eds.): Essays on Language Function and Language Type, Amsterdam: John Benjamins, 437-467. SLobin, Dan, 2004: "How people move" en Carol Lynn Moder y Aida Martinovic-Zic (eds.): Discourse Across Languages and Cultures, Philadelphia: John Benjamins, 195-210.

Von Stutterheim, Christiane, 2003: "Linguistic structure and information organization. The case of very advanced learners" en S. Foster-Cohen (ed.): EuroSLA Yearbook, Amsterdam: John Benjamins, 183-206.

Von Stutterheim, Christiane y Ralph Nüse, 2003: "Processes of conceptualization in language production: Language-specific perspectives and event construal”, Linguistics 41(5), 851-881.

TALmy, Leonard, 1985: “Lexicalization patterns: Semantic structure in lexical forms” en Timothy SHOPEN (ed.): Language typology and syntactic description, vol. 3: Grammatical categories and 
the lexicon, Cambridge: Cambridge University Press.

TALmy, Leonard, 2000: Towards a cognitive semantics, Cambridge, MA: MIT Press.

TEGELBERG, Elisabeth, 2002: "Traducteurs et lexicographes face à la problematique des verbes de mouvement du suédois et du français”, Studia Neophilologica 74, 180-206.

Treffers-Daller, Jeanine, 2012: "Thinking for speaking and linguistic relativity among bilinguals: towards a new research agenda”, Language, Interaction and Acquisition 3(2), 288-300.

Treffers-Daller, Jeanine y Jeanette Sakel, 2012: "Why transfer is a key aspect of language use and processing in bilinguals and L2-users", International Journal of Bilingualism 16(1), 3-10.

ZubizarRetA, María Luisa y Eunjeong OH, 2007: On the syntactic composition of manner and motion, Cambridge, Mass: MIT Press. 